TITLE:

\title{
Energy-Based Magnetization and Magnetostriction Modeling of Grain-Oriented Silicon Steel under Vectorial Excitations
}

\section{$\operatorname{AUTHOR}(S)$ :}

Ito, S.; Mifune, T.; Matsuo, T.; Kaido, C.

\section{CITATION:}

Ito, S. ... [et al]. Energy-Based Magnetization and Magnetostriction Modeling of GrainOriented Silicon Steel under Vectorial Excitations. IEEE Transactions on Magnetics 2016, 52(5): 7386682.

\section{ISSUE DATE:}

2016-05-01

URL:

http://hdl.handle.net/2433/217020

\section{RIGHT:}

(C) 2016 IEEE. Personal use of this material is permitted. Permission from IEEE must be obtained for all other uses, in any current or future media, including reprinting/republishing this material for advertising or promotional purposes, creating new collective works, for resale or redistribution to servers or lists, or reuse of any copyrighted component of this work in other works. 


\title{
Energy-based magnetization and magnetostriction modeling of grain-oriented silicon steel under vectorial excitations
}

\author{
S. Ito ${ }^{1}, \mathrm{~T}$. Mifune ${ }^{1}$, Member IEEE, T. Matsuo ${ }^{1}$, Member IEEE, and C. Kaido ${ }^{2}$, \\ ${ }^{1}$ Kyoto University, Kyotodaigaku-katsura, Nishikyo-ku, Kyoto, 615-8510, Japan. \\ ${ }^{2}$ Kitakyushu National College of Technology, 5-20-1 Shii, Kokuraminamiku, Kitakyushu, Fukuoka, 802-0985, \\ Japan.
}

\begin{abstract}
The assembled domain structure model (ADSM), is an energy-based macroscopic magnetization model of a core material. In this study, we used the ADSM to simulate the magnetization curves and magnetostriction of a GO silicon steel sheet under vectorial excitations and we measured the same sheet using the rotational single sheet tester with a three-element strain gage rosette. In the simulation, the target value of the average magnetization vector representing unidirectional or rotational magnetic flux is given as a known parameter. We obtained the magnetization state by finding the external magnetic field vector and state vectors, satisfying the criteria that the average magnetization vector equals the target value and that the total magnetic energy is locally minimized. The simulation reconstructed the magnetostriction produced by $90^{\circ}$ domain-wall motion under unidirectional magnetization as well as the vectorial contraction and extension under rotational magnetization.
\end{abstract}

Index Terms—cubic anisotropy, multiscale model, silicon steel sheet, magnetostriction.

\section{INTRODUCTION}

$\mathrm{T}$ HE magnetic and mechanical behaviors of iron-core materials are strongly coupled, and mechanical stress affects magnetization properties. Magnetic properties are also affected by magnetostriction [1] induced by magnetization processes [2][3] such as magnetic domain-wall motion and magnetization rotation. Magnetostriction is also a cause of noise and vibration. To predict the magnetic behaviors of core materials, it is important to understand these magnetocoupling effects. So far, to model the macroscopic magnetization property, several hysteresis models such as the vector Preisach model [8] and the vector play model [9] have been developed. Most of these models are phenomenologically constructed based on the parameter fitting to measured magnetization properties. However, to extend these models taking magneto-coupling effects into account, magneto-mechanically coupled measurements are required with a vast set of magnetic and mechanical parameters.

Several physical models have been proposed to predict macroscopic magnetization property from material constants without using measured BH loops. A multiscale model of reversible magneto-elastic behavior assuming magnetic domain equilibrium based on the probability function is presented in [4]. This model successfully reconstructed the magnetization curves and magnetostriction of grain-oriented (GO) silicon steel sheet [5]. Ito et al. [6] proposed a multiscale model called the assembled domain structure model (ADSM) constituted by the assembly of mesoscopic six-domain magnetic particles. The ADSM is a macroscopic model based on local minimization of total magnetic energy to represent the hysteretic behavior of a magnetic sheet. However, this model only simulated unidirectional magnetization, and the magnetostriction was not compared with measured results.

In this study, using the ADSM we simulate the magnetization and magnetostriction of GO under vectorial excitations, then compare the simulated properties with properties measured using a rotational single-sheet tester with strain gages.

\section{MEASUREMENT OF MAGNETIZATION CURVES AND MAGNETOSTRICTION}

The magnetization curves and magnetostriction were measured with a rotational single-sheet tester, using a stator of a two-pole induction motor with strain gages. The circular sample was magnetized by two exciting coils, placed along the $x$ - and $y$-directions. The excitation currents were controlled to set the magnetic flux density to be unidirectional or rotational. Magnetostriction at angles of $0^{\circ}, 45^{\circ}$, and $90^{\circ}$ from the rolling direction (RD) was measured on a three-element strain gage rosette. The magnetic flux density and magnetic field were measured on the $B$-coil and $H$-coil, respectively.

The diameter of the sample was $7.6 \mathrm{~cm}$, and the thickness was $0.3 \mathrm{~mm}$. The width of the $B$-coil and $H$-coil were $3.8 \mathrm{~cm}$. The distance between the sample and magnetic shield was 0.5 $\mathrm{cm}$.

\section{ASSEMBLED DOMAIN STRUCTURE MODEL}

\section{A. SDSM and ADSM}

The ADSM, a way to describe macroscopic magnetization properties, is the assembly of simplified domain structure models (SDSMs) (Fig. 1). Each SDSM is regarded as a cell of the ADSM. The SDSM has six domains, where magnetization vectors in six domains are directed nearly parallel/antiparallel to the three easy axes of cubic anisotropy. The magnetization state of each cell is represented by the state vector $x(j)=\left(\theta_{1}\right.$, $\left.\ldots, \theta_{6}, \phi_{1}, \ldots, \phi_{6}, r_{1}, \ldots, r_{5}\right)$, where $j$ is the cell index. The variables $r_{\mathrm{i}}$ are the volume ratios of the domains, where $r_{6}=1$ $-r_{1}-r_{2}-r_{3}-r_{4}-r_{5}$. The normalized magnetization vectors $\left(\boldsymbol{m}_{1}, \ldots, \boldsymbol{m}_{6}\right)$ is given by $\boldsymbol{m}_{\mathrm{i}}=\left(\sin \theta_{\mathrm{i}} \cos \phi_{\mathrm{i}}, \sin \theta_{\mathrm{i}} \sin \phi_{\mathrm{i}}, \cos \theta_{\mathrm{i}}\right)$, where $i$ is the index of the domains. The entire magnetization states are represented by the variable vector $\boldsymbol{X}$, consisting of $x(j)(j=1, \ldots)$ in each cell. 


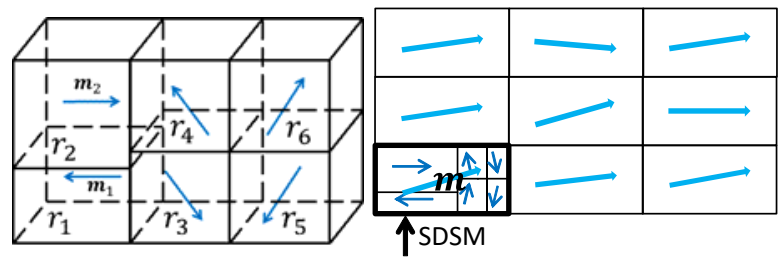

(a) Simplified domain structure model (b)Assembled domain structure model

Fig. 1. Assembled domain structure model.

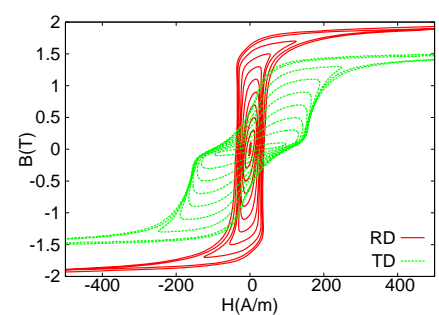

Fig. 2. Measured BH loops along RD and TD.

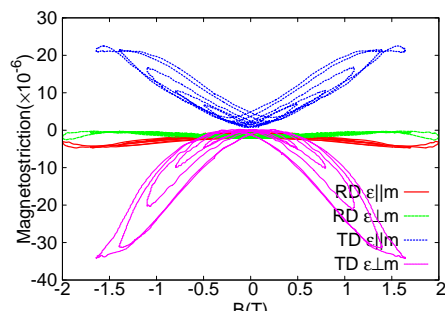

Fig. 3. Measured magnetostriction along RD and TD.

The vector $\boldsymbol{X}$ is determined so as to locally minimize the normalized total magnetic energy $e$. The total normalized energy is the sum of the Zeeman energy, the crystalline anisotropic energy, the magnetostatic energy, and the magnetoelastic energy [see Appendix].

The ADSM assumes that the pinning effect, caused by pinning sites such as crystal defects and crystal grain boundaries, is represented by a uniform pinning field. The uniform pinning field is represented using the vector stop hysteron [7]. Accounting for the pinning field similarly to the demagnetization field, the effect of the pinning field is added to $\partial e / \partial \boldsymbol{X}$ as $-\boldsymbol{h}_{\mathrm{p}} \partial \boldsymbol{m}(j) / \partial \boldsymbol{x}(j)$, where $\boldsymbol{h}_{\mathrm{p}}$ is the pinning field normalized by the anisotropy field and $\boldsymbol{m}(j)$ is the average magnetization vector of the cell $j$. The magnetization states are obtained by solving $\partial e / \partial \boldsymbol{X}=0$.

Sudo et al. [7] obtained a local minimum by numerically integrating an artificial state equation given as

$\mathrm{d} \boldsymbol{X} / \mathrm{d} t=\boldsymbol{Y}, \mathrm{d} \boldsymbol{Y} / \mathrm{d} t=-\partial e / \partial \boldsymbol{X}-\beta \boldsymbol{Y}$

until reaching the steady state, where $\boldsymbol{Y}$ is an intermediate variable vector and $\beta$ is a damping coefficient. Equation (1) can be interpreted as the system of equations of motion where the inertia and the damping factors for the motion of magnetization vectors and domain walls are artificially given to obtain a stable equilibrium in a simple way.

In the simulation under vectorial excitations, the target value of the average normalized magnetization vector $\boldsymbol{m}_{\mathrm{a}}=\left(m_{\mathrm{ax}}, m_{\mathrm{ay}}\right.$, 0 ) representing unidirectional or rotational magnetic flux is given as a known parameter. Under rotational excitation, for example, $\boldsymbol{m}_{\mathrm{a}}$ is set as $\left(m_{\mathrm{a}} \cos \theta, m_{\mathrm{a}} \sin \theta, 0\right)$, where $m_{\mathrm{a}}$ is constant

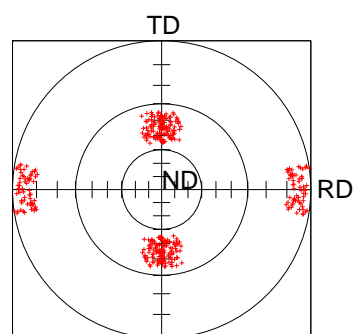

Fig. 4. $<100>$ pole figure of the crystal orientation used in the simulation. (ND is an abbreviation of the normal direction.)

and $\theta$ changes from 0 to $2 \pi$. The magnetization state is obtained by finding the variable vector $\boldsymbol{X}$ and the normalized external magnetic field $\boldsymbol{h}=\left(h_{\mathrm{x}}, h_{\mathrm{y}}, 0\right)$, satisfying $\boldsymbol{m}_{\mathrm{ave}}=\boldsymbol{m}_{\mathrm{a}}$ and $\partial e / \partial \boldsymbol{X}=0$, where $\boldsymbol{m}_{\mathrm{ave}}$ is the average normalized magnetization vector of all cells. We obtained the magnetization state by numerically integrating (1) and another artificial state equation given as

$$
\begin{aligned}
& \mathrm{d} h_{\mathrm{x}} / \mathrm{d} t=g_{\mathrm{x}}, \mathrm{d} h_{\mathrm{y}} / \mathrm{d} t=g_{\mathrm{y}}, \\
& \mathrm{d} g_{\mathrm{x}} / \mathrm{d} t=R\left(m_{\mathrm{ax}}-m_{\text {avex }}\right)-\beta_{\mathrm{h}} g_{\mathrm{x}}, \\
& \mathrm{d} g_{\mathrm{y}} / \mathrm{d} t=R\left(m_{\text {ay }}-m_{\text {avey }}\right)-\beta_{\mathrm{h}} g_{\mathrm{y}},
\end{aligned}
$$

where $R$ is a constant, $g_{\mathrm{x}}$, and $g_{\mathrm{y}}$ are intermediate variables, and $\beta_{\mathrm{h}}$ is a dissipation coefficient.

\section{B. Calculation of magnetostriction}

The components of the magnetostriction tensor $\varepsilon^{\prime}$ with respect to the cubic crystal system are obtained as

$$
\varepsilon^{\prime}=\frac{3}{2}\left(\begin{array}{ccc}
\lambda_{100}\left(\alpha_{1, i}{ }^{2}-\frac{1}{3}\right) & \lambda_{111} \alpha_{1, i} \alpha_{2, i} & \lambda_{111} \alpha_{1, i} \alpha_{3, i} \\
\lambda_{111} \alpha_{1, i} \alpha_{2, i} & \lambda_{100}\left(\alpha_{2, i}{ }^{2}-\frac{1}{3}\right) & \lambda_{111} \alpha_{2, i} \alpha_{3, i} \\
\lambda_{111} \alpha_{1, i} \alpha_{3, i} & \lambda_{111} \alpha_{2, i} \alpha_{3, i} & \lambda_{100}\left(\alpha_{3, i}{ }^{2}-\frac{1}{3}\right)
\end{array}\right),
$$

where $\left(\alpha_{1, i}, \alpha_{2, i}, \alpha_{3, i}\right)$ is the direction cosine of the magnetization vectors of domain $i$ with respect to the three easy axes. By transforming the coordinates from the coordinate system based on crystal orientation to one with coordinate axes along the RD, the transverse direction (TD), and the thickness direction, we obtain the magnetostriction tensor $\varepsilon$ with respect to the coordinate system based on the RD and TD.

The total magnetostriction in the material is calculated by

$$
\varepsilon_{\text {global }}=\frac{1}{N} \sum_{j}^{\mathrm{N}} \sum_{i}^{6} r_{i} \varepsilon,
$$

where $N$ is the number of cells.

\section{MEASURED AND SIMULATED RESUlTS}

\section{A. Results along the RD and TD}

Figures 2 and 3 show the measured magnetization curves and magnetostriction curves of the low-grade GO silicon steel along the $\mathrm{RD}$ and TD. Magnetostriction parallel to the magnetization direction $(\varepsilon \| m)$ and perpendicular to the magnetization direction are portrayed in Fig. 3. Large magnetostriction appeared under magnetization along the TD, while it was very small under magnetization along the RD. 


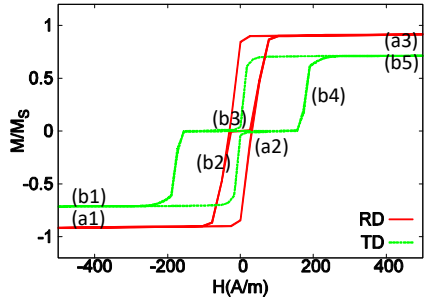

Fig. 5. Simulated BH loops along RD and TD.

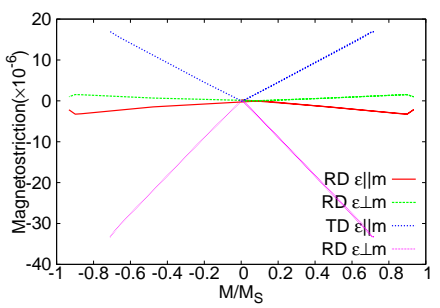

Fig. 6. Simulated magnetostriction along RD and TD.

(a)
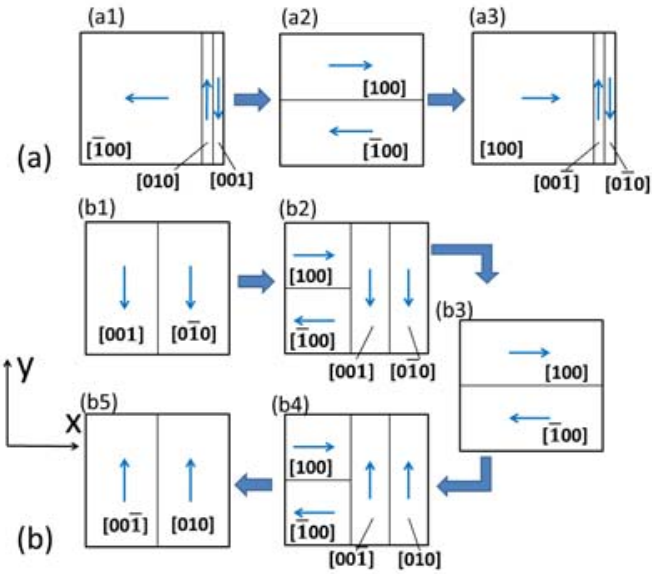

Fig. 7. Magnetization process of a SDSM cell : (a) RD and (b) TD.

Figure 4 shows a pole figure of the crystal orientation used in the simulation, where $<100\rangle$ directions of all the cells are plotted. The easy axes are assumed to be distributed within $10^{\circ}$. Figures 5 and 6 show simulated magnetization curves and magnetostriction curves with the anisotropy constant $K=$ $3.8 \times 10^{4} \mathrm{Jm}^{-3}$, along with the magnetostriction constants $\lambda_{100}=$ $2.3 \times 10^{-5}, \lambda_{111}=-5.0 \times 10^{-6}, \mu_{0} M_{\mathrm{S}}=2.2 \mathrm{~T}$. We assume a tensile stress of $4 \mathrm{MPa}$, induced by the insulation coating, along the $\mathrm{RD}$. The radius of the vector stop hysteron is 0.01 , and the magnitudes of the pinning field along the RD and TD are $2.0 \times 10^{-3}$ and $5.0 \times 10^{-3}$, respectively. $8 \times 8 \times 1$ cells are used where the cell has a dimension ration of $1: 1: 10^{-4}$.

Figure 7 portrays the simulated magnetization process of a SDSM cell of the material, which represents the volume ratios of the domains only schematically, and its domain boundaries do not reflect real domain walls.

Magnetization along the RD is produced mainly by $180^{\circ}$ domain-wall motion. In this cell, there is a small submergence angle between the [100] direction and the surface of sheet.
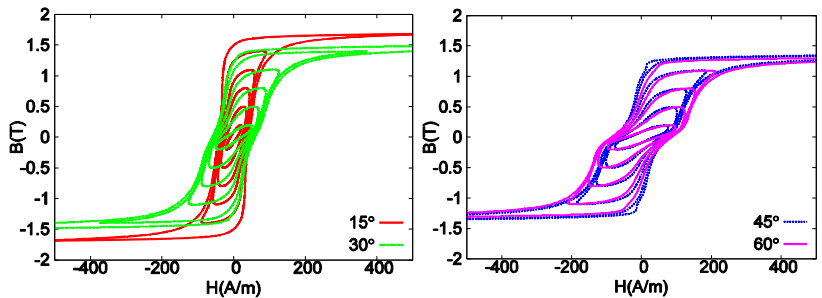

Fig. 8. Measured BH loops under alternating magnetic flux.
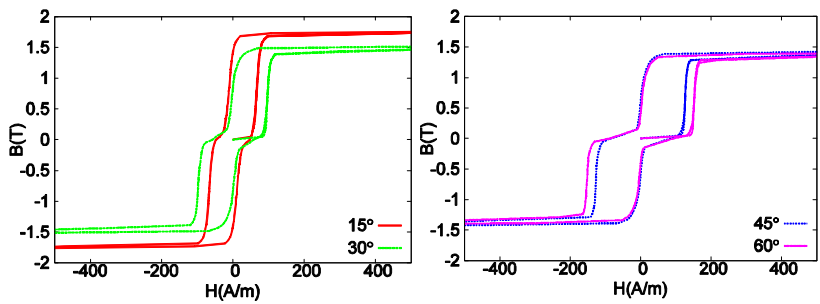

Fig. 9. Simulated $\mathrm{BH}$ loops under alternating magnetic flux.

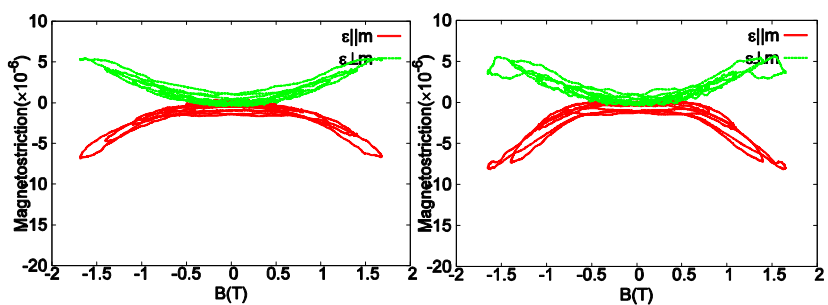

(a) $15^{\circ}$

(b) 30

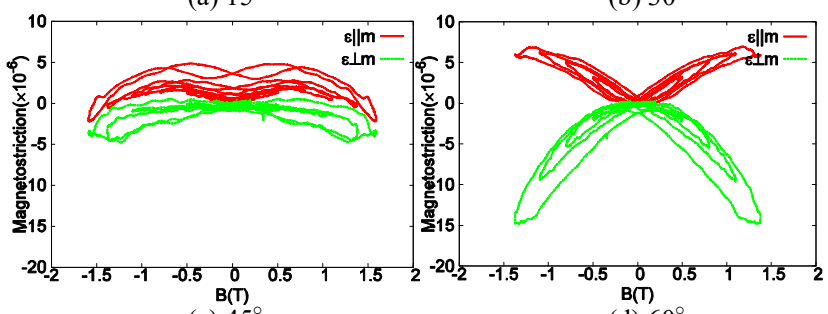

(c) $45^{\circ}$

(d) $60^{\circ}$

Fig. 10. Measured magnetostriction under alternating magnetic flux.

Accordingly, magnetic poles appear on the surface of the sheet when the cell is magnetized along the RD by $180^{\circ}$ domainwall motion. To cancel these magnetic poles, domains form that are magnetized along the [001] and [010] directions. These lancet domains reduce the magnetostriction along the $\mathrm{RD}$. The increase in magnetostriction under high magnetic flux is caused by the magnetization rotation.

Magnetization along the TD is produced mainly by the $90^{\circ}$ domain-wall motion. Accordingly, large magnetostriction appears.

\section{B. Results along the intermediate directions}

Figures 8 and 9 show the measured and simulated magnetization curves along the directions angled $15^{\circ}, 30^{\circ}, 45^{\circ}$, and $60^{\circ}$ from the RD. At applied fields over $300 \mathrm{~A} / \mathrm{m}$, the magnetic flux density saturates. This magnetic flux density is high along the $0^{\circ}$ direction and low along the $60^{\circ}$ direction, because the RD is close to the [100] direction and because the $55^{\circ}$ direction from the RD is close to the [111] direction. 


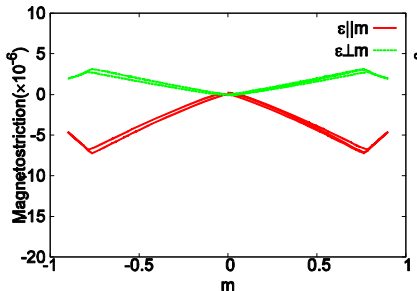

(a) $15^{\circ}$

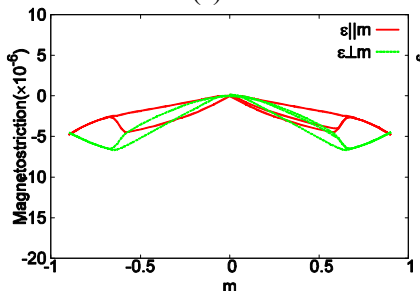

(c) $45^{\circ}$

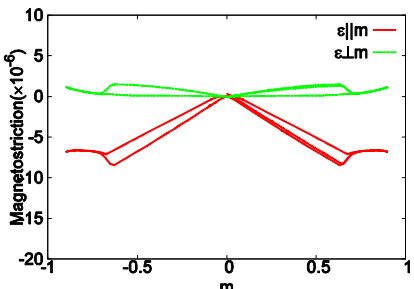

(b) $30^{\circ}$

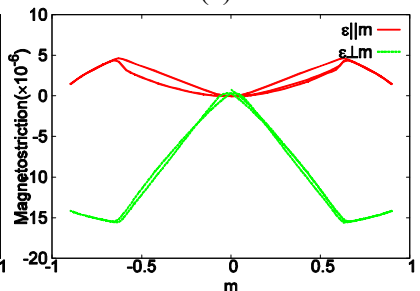

(d) $60^{\circ}$
Fig. 11. Simulated magnetostriction under alternating magnetic flux.

Figures 10 and 11 show the measured and simulated magnetostriction. Under magnetization along the $15^{\circ}, 30^{\circ}, 45^{\circ}$ directions, the magnetostriction is small because the magnetization is produced mainly by $180^{\circ}$ domain-wall motion. Under magnetization along the $60^{\circ}$ direction, the magnetostriction is large because the magnetization is produced mainly by $90^{\circ}$ domain-wall motion.

\section{Results under the rotational magnetization condition}

Figures 12 shows the measured and simulated loci of the $H$ vector under the rotational magnetization condition, with a magnetic flux density of $1.5,1.6$, or $1.7 \mathrm{~T}$. To magnetize along the $55^{\circ}$ direction, a large magnetic field is needed, while along the $0^{\circ}$ direction only a very small field is needed.

Figures 13 shows the measured and simulated loci of principal strain of magnetostriction. The lines $\varepsilon(+)$ and $\varepsilon(-)$ are the tensile strain and compressive strain, respectively. The GO silicon steel sheet was contracted and extended along the RD and TD, respectively. The simulated magnetic properties agree well with the measured properties.

\section{CONCLUSION}

Using the ADSM, we simulated magnetization curves and magnetostriction under alternating magnetic flux along several directions and under rotational magnetization. The simulated properties agreed well with the measured properties.

\section{APPENDIX}

The normalized total magnetic energy $e$ consists of the Zeeman energy, the crystalline anisotropic energy, the magnetostatic energy, and the magnetoelastic energy [6]. Normalized energy components in domain $i$ are given as $-2 r_{\mathrm{i}} \boldsymbol{h} \cdot \boldsymbol{m}_{\mathrm{i}}, \quad r_{\mathrm{i}}\left(\alpha_{1, \mathrm{i}}{ }^{2} \alpha_{2, \mathrm{i}}{ }^{2}+\alpha_{2, \mathrm{i}}{ }^{2} \alpha_{3, \mathrm{i}}{ }^{2}+\alpha_{3, \mathrm{i}}{ }^{2} \alpha_{1, \mathrm{i}}{ }^{2}\right),-r_{\mathrm{i}} \boldsymbol{h}_{\mathrm{st}} \cdot \boldsymbol{m}_{\mathrm{i}}$, and $-r_{\mathrm{i}} \sigma: \varepsilon / K$, respectively, where $\boldsymbol{h}$ and $\boldsymbol{h}_{\text {st }}$ are the applied and demagnetizing fields normalized by the anisotropy field, $\sigma$ is the applied stress tensor, and $K$ is the anisotropy constant. The demagnetizing field is given by the convolution of magnetization vectors and demagnetization coefficients [7].
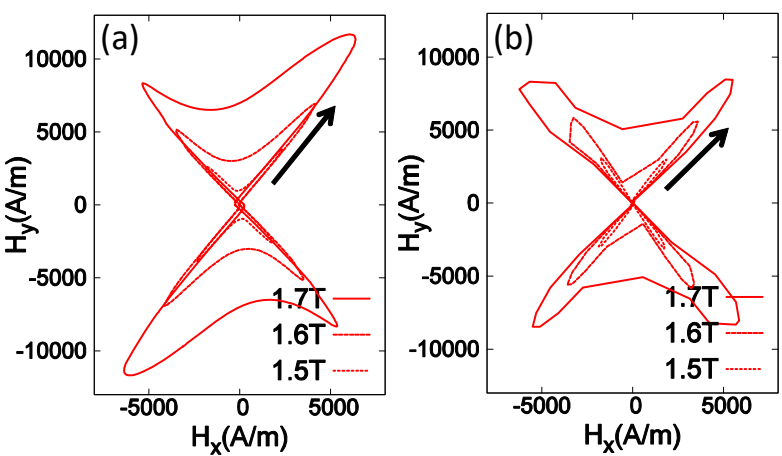

Fig. 12. Loci of $\mathrm{H}$ vector : (a) measured (b) simulated.

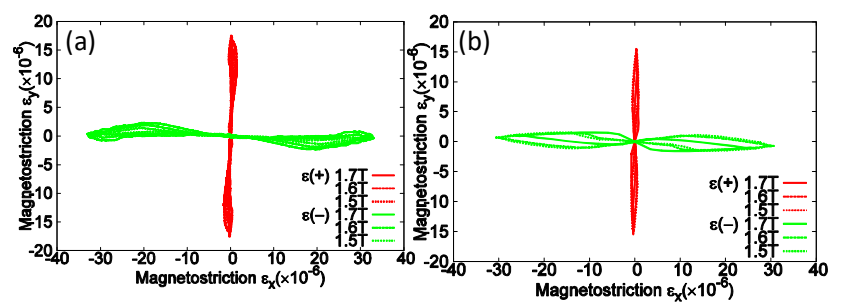

Fig. 13. Loci of principal strain of magnetostriction : (a) measured (b) simulated.

It is known that the pinning field can be modeled by the play hysteron for the input of magnetic field [10]. Accordingly it is rewritten with the stop hysteron for the input of magnetization (or domain wall position). In this study, its magnitude is determined based on the measured coercive field. More details are explained in [6].

\section{ACKNOWLEDGMENT}

This work was supported in part by the Japan Society for the Promotion of Science under a Grant-in-Aid for Scientific Research (C), Grant No. 26420232.

\section{REFERENCES}

[1] A. J. Moses, P. I. Anderson, and S. Somkun, "Modeling 2-D magnetostriction in nonoriented electrical steels using a simple magnetic domain model," IEEE. Trans. Magn., vol. 51, 6000407.

[2] A. Hubert, and R. Schäfer, Magnetic Domains: The Analysis of Magnetic Microstructures, Berlin Heidelberg : Springer-Verlag, 1998.

[3] H. Kronmüeller and M. Fähnle, Micromagnetism and the Microstructure of Ferromagnetic Solids, Cambridge: Cambridge University Press, 2003.

[4] L. Daniel, O. Hubert, N. Buiron, and R. Billardon, "Reversible magnetoelastic behavior: A multiscale approach," J. Mech. Phys. Solids., vol. 56, pp. 1018-1042.

[5] O. Hubert and L. Daniel, "Multiscale modeling of the magnetomechanical behavior of grain-oriented silicon steels," J. Magn. Magn. Mater., vol. 320, pp. 1412-1422.

[6] S. Ito, T. Mifune, T. Matsuo, and C. Kaido, "Macroscopic magnetization modeling of silicon steel sheets using an assembly of six-domain particles," J. Appl. Phys., vol. 117, 17D126.

[7] M. Sudo, and T. Matsuo, "Magnetization modeling of silicon steel using a simplified domain structure model," J. Appl. Phys., vol. 111, $07 \mathrm{D} 107$.

[8] E. Cardelli, E. D. Torre, and A. Faba, "A general vector hysteresis operator: extension to the 3-D case," IEEE. Trans. Magn., vol. 46, pp. 3990-4000.

[9] T. Matsuo, "An anisotropic vector hysteresis model using isotropic vector play model," IEEE. Trans. Magn., vol. 46, pp. 3041-3044.

[10] A. Bergqvist, "Magnetic vector hysteresis model with dry friction-like pinning," Physica B, vol. 233, pp. 342-347. 